\title{
THE PAIR CORRELATION OF ZEROS OF THE ZETA FUNCTION
}

\author{
H. L. MONTGOMERY
}

1. Statement of results. We assume the Riemann Hypothesis $(\mathrm{RH})$ throughout this paper; $\varrho=\frac{1}{2}+i \gamma$ denotes a nontrivial zero of the Riemann zeta function. Our object is to investigate the distribution of the differences $\gamma-\gamma^{\prime}$ between the zeros. It would thus be desirable to know the Fourier transform of the distribution function of the numbers $\gamma-\gamma^{\prime}$; with this in mind we take

$$
F(\alpha)=F(\alpha, T)=\left(\frac{T}{2 \pi} \log T\right)^{-1} \sum_{0<\gamma \leqq T ; 0<\gamma^{\prime} \leqq T} T^{i \alpha\left(\gamma-\gamma^{\prime}\right)} w\left(\gamma-\gamma^{\prime}\right),
$$

where $\alpha$ and $T \geqq 2$ are real. Here $w(u)$ is a suitable weighting function, $w(u)=4 /\left(4+u^{2}\right)$, so $w(0)=1$. Our results concerning $F(\alpha)$ are stated in the following

Theorem. (Assume RH.) For real $\alpha, T \geqq 2$, let $F(\alpha)$ be defined by (1). Then $F(\alpha)$ is real, and $F(\alpha)=F(-\alpha)$. If $T>T_{0}(\varepsilon)$ then $F(\alpha) \geqq-\varepsilon$ for all $\alpha$. For fixed $\alpha$ satisfying $0 \leqq \alpha<1$ we have

$$
F(\alpha)=(1+o(1)) T^{-2 \alpha} \log T+\alpha+o(1)
$$

as $T$ tends to infinity; this holds uniformly for $0 \leqq \alpha \leqq 1-\varepsilon$.

The first term on the right-hand side of the above behaves in the limit as a Dirac $\delta$-function; it reflects the fact that if $\alpha=0$ then all the terms in (1) are positive. With more effort we could show that (2) holds uniformly throughout $0 \leqq \alpha \leqq 1$.

To investigate sums involving $\gamma-\gamma^{\prime}$ we have only to convolve $F(\alpha)$ with an 
appropriate kernel $\hat{r}(\alpha)$; from (1) alone it is immediate that

$$
\sum_{0<\gamma \leqq T ; 0<\gamma^{\prime} \leqq T} r\left(\left(\gamma-\gamma^{\prime}\right) \frac{\log T}{2 \pi}\right) w\left(\gamma-\gamma^{\prime}\right)=\left(\frac{T}{2 \pi} \log T\right) \int_{-\infty}^{+\infty} F(\alpha) \hat{r}(\alpha) d \alpha .
$$

Here $\hat{r}$ is the Fourier transform of $r$,

$$
\hat{r}(\alpha)=\int_{-\infty}^{+\infty} r(u) e(-\alpha u) d u \quad\left(e(\theta)=e^{2 \pi i \theta}\right)
$$

Our theorem gives us little information about $F(\alpha)$ for $\alpha \geqq 1$, so for the most part we restrict our attention to kernels $\hat{r}$ which vanish outside $[-1+\delta, 1-\delta]$. Particular choices of $\hat{r}(\alpha)$ give us

Corollary 1. (Assume RH.) If $0<\alpha<1$ is fixed then

$$
\sum_{0<\gamma \leqq T ; 0<\gamma^{\prime} \leqq T}\left(\frac{\sin \alpha\left(\gamma-\gamma^{\prime}\right) \log T}{\alpha\left(\gamma-\gamma^{\prime}\right) \log T}\right) w\left(\gamma-\gamma^{\prime}\right) \sim\left(\frac{1}{2 \alpha}+\frac{\alpha}{2}\right) \frac{T}{2 \pi} \log T,
$$

and

(6)

$$
\sum_{0<\gamma \leqq T ; 0<\gamma^{\prime} \leqq}\left(\frac{\sin (\alpha / 2)\left(\gamma-\gamma^{\prime}\right) \log T}{(\alpha / 2)\left(\gamma-\gamma^{\prime}\right) \log T}\right)^{2} w\left(\gamma-\gamma^{\prime}\right) \sim\left(\frac{1}{\alpha}+\frac{\alpha}{3}\right) \frac{T}{2 \pi} \log T
$$

In the latter assertion one can delete the factor $w\left(\gamma-\gamma^{\prime}\right)$ if one wishes. We use (6) to derive

\section{COROllary 2. (Assume RH.) As $T$ tends to infinity}

$$
\sum_{0<\gamma \leqq T ; \varrho \text { simple }} 1 \geqq\left(\frac{2}{3}+o(1)\right) \frac{T}{2 \pi} \log T .
$$

The number of zeros of $\zeta(s)$ with $0<\gamma \leqq T$ is $\sim(T / 2 \pi) \log T$, so the above asserts that at least $\frac{2}{3}$ of the zeros are simple. It is known (see [6]) that the first $3,500,000$ zeros are simple and lie on the critical line $\sigma=\frac{1}{2}$. Although one expects that all the zeros of $\zeta(s)$ are simple, the only other result in this direction is due to A. Selberg [7]. His result holds unconditionally; it states that a positive density of the zeros of $\zeta(s)$ are of odd order and lie on the critical line.

Let $0<\gamma_{1} \leqq \gamma_{2} \leqq \ldots$ denote the imaginary parts of the zeros of $\zeta(s)$ in the upper 
half-plane. The average of $\gamma_{n+1}-\gamma_{n}$ is $2 \pi / \log \gamma_{n}$; our Theorem enables us to show that $\gamma_{n+1}-\gamma_{n}$ is not always near its average.

COROllary 3. (Assume RH.) We can compute a constant $\lambda$ so that

$$
\lim _{n} \inf \left(\gamma_{n+1}-\gamma_{n}\right)\left(\log \gamma_{n} / 2 \pi\right) \leqq \lambda<1
$$

A complicated argument would permit one to show that in fact $\gamma_{n+1}-\gamma_{n} \leqq$ $2 \pi \lambda / \log \gamma_{n}$ for a positive density of $n$. This, with the fact that the average value is $2 \pi / \log \gamma_{n}$, enables one to assert that

$$
\lim \sup _{n}\left(\gamma_{n+1}-\gamma_{n}\right)\left(\log \gamma_{n} / 2 \pi\right) \geqq \lambda^{\prime}>1
$$

We note that if $\zeta(s)$ has infinitely many multiple zeros then we may take $\lambda=0$ in (8). Our proof allows us to take $\lambda=0.68$. It would be of interest to have $\lambda<\frac{1}{4}$, as P. J. Weinberger and I have established the following: Let $d>0$ be square-free, and put $K=Q\left((-d)^{1 / 2}\right)$. Let $h(-d)$ be the class number of $K$, and let $\zeta_{K}(s)=\zeta(s)$ - $L(s, \chi)$ be the Dedekind zeta function of $K$. For each positive $A, \varepsilon$ there is an effectively computable constant $d_{0}=d_{0}(A, \varepsilon)$ such that if $h(-d) \leqq A, d>d_{0}$, then all zeros of $\zeta_{K}(s)$ which are in the rectangle $0<\sigma<1,0 \leqq t \leqq d^{1 / 2-\varepsilon}$ lie on the line $\sigma=\frac{1}{2}$; if $\frac{1}{2}+i \gamma_{n}, \frac{1}{2}+i \gamma_{n+1}$ are consecutive zeros of $\zeta_{K}(s)$ in this range then

$$
(1-\varepsilon) \frac{2 \pi}{\log d\left(\gamma_{n}+2\right)^{2}} \leqq \gamma_{n+1}-\gamma_{n} \leqq(1+\varepsilon) \frac{2 \pi}{\log d\left(\gamma_{n}+2\right)^{2}}
$$

One may inquire about the behaviour of $F(\alpha)$ for $\alpha \geqq 1$. Our first observation is that (2) cannot hold uniformly for $0 \leqq \alpha \leqq C$ if $C$ is large. For if it did then (6) would hold for $0<\alpha \leqq C$. Write $(6)$ as $G(\alpha) \sim H(\alpha)$. On one hand $|\sin 2 x| \leqq 2|\sin x|$, so $G(2 \alpha) \leqq G(\alpha)$ for all $\alpha$. On the other hand $H(2 \alpha)>\frac{3}{2} H(\alpha)$ for $\alpha \geqq 2$. This suggests that $F(\alpha)$ makes some change in its behaviour for $\alpha \geqq 1$. Further considerations of the above sort lead one to believe that certain averages of $F(\alpha)$ over large $\alpha$ are close to 1 . At the end of $\S 3$ we describe two heuristic arguments which suggest that

$$
F(\alpha)=1+o(1)
$$

for $\alpha \geqq 1$, uniformly in bounded intervals. This, with the Theorem, completely determines $F$, so an appropriate use of (3) leads immediately to a

CONJECTURE. For fixed $\alpha<\beta$, 


$$
1 \sim\left(\int_{\alpha}^{\beta} 1-\left(\frac{\sin \pi u}{\pi u}\right)^{2} d u+\delta(\alpha, \beta)\right) \frac{T}{2 \pi} \log T
$$

as $T$ tends to infinity. Here $\delta(\alpha, \beta)=1$ if $0 \in[\alpha, \beta], \delta(\alpha, \beta)=0$ otherwise.

The Dirac $\delta$-function occurs naturally in the above, for if $0 \in[\alpha, \beta]$ then the sum includes terms $\gamma=\gamma^{\prime}$.

The assertions (11) and (12) are essentially equivalent. From either it immediately follows that almost all zeros are simple. From (11) it is easy to see how Corollary 1 ought to be extended: If $(11)$ is true then for $\alpha \geqq 1$,

$$
\sum_{0<\gamma \leqq T ; 0<\gamma^{\prime} \leqq T}\left(\frac{\sin \alpha\left(\gamma-\gamma^{\prime}\right) \log T}{\alpha\left(\gamma-\gamma^{\prime}\right) \log T}\right) w\left(\gamma-\gamma^{\prime}\right) \sim \frac{T}{2 \pi} \log T
$$

and

$$
\sum_{0<\gamma \leqq T ; 0<\gamma^{\prime} \leqq T}\left(\frac{\sin (\alpha / 2)\left(\gamma-\gamma^{\prime}\right) \log T}{(\alpha / 2)\left(\gamma-\gamma^{\prime}\right) \log T}\right)^{2} w\left(\gamma-\gamma^{\prime}\right) \sim\left(1+\frac{1}{3 \alpha^{2}}\right) \frac{T}{2 \pi} \log T .
$$

In a certain standard terminology the Conjecture may be formulated as the assertion that $1-((\sin \pi u) / \pi u)^{2}$ is the pair correlation function of the zeros of the zeta function. F. J. Dyson has drawn my attention to the fact that the eigenvalues of a random complex Hermitian or unitary matrix of large order have precisely the same pair correlation function (see [3, equations (6.13), (9.61)]). This means that the Conjecture fits well with the view that there is a linear operator (not yet discovered) whose eigenvalues characterize the zeros of the zeta function. The eigenvalues of a random real symmetric matrix of large order have a different pair correlation, and the eigenvalues of a random symplectic matrix of large order have yet another pair correlation. In fact the "form factors" $F_{r}(\alpha), F_{s}(\alpha)$ of these latter pair correlations are nonlinear for $0<\alpha<1$, so our Theorem enables us to distinguish the behaviour of the zeros of $\zeta(s)$ from the eigenvalues of such matrices. Hence, if there is a linear operator whose eigenvalues characterize the zeros of the zeta function, we might expect that it is complex Hermitian or unitary.

One might extend the present work to investigate the $k$-tuple correlation of the zeros of the zeta function. If the analogy with random complex Hermitian matrices appears to continue, then one might conjecture that the $k$-tuple correlation function $\hat{F}\left(u_{1}, u_{2}, \ldots, u_{k}\right)$ is given by

$$
\hat{F}\left(u_{1}, u_{2}, \ldots, u_{k}\right)=\operatorname{det} A \text {, }
$$


where $A=\left[a_{i j}\right]$ is the $k \times k$ matrix with entries $a_{i i}=1, a_{i j}=\left(\sin \pi\left(u_{i}-u_{j}\right)\right) / \pi\left(u_{i}-u_{j}\right)$ for $i \neq j$. Here the normalization is the same as in the Conjecture, which is the case $k=2$ of the above.

If one continues to draw on the analogy with random complex Hermitian matrices then one may formulate a conjecture concerning the distribution of the numbers $\gamma_{n+1}-\gamma_{n}$. The precise conjecture involves a complicated (but calculable) spheroidal function. Thus, or otherwise, one may conjecture that

$$
\lim _{n} \inf \left(\gamma_{n+1}-\gamma_{n}\right) \log \gamma_{n}=0
$$

and

$$
\lim \sup \left(\gamma_{n+1}-\gamma_{n}\right) \log \gamma_{n}=+\infty
$$

so Corollary 3 is probably far from the truth.

It would be interesting to see how numerical evidence compares with the above conjectures. The first several thousand zeros have been computed, so it would not be difficult to assemble relevant statistics. However, data on the failures of "Gram's law" indicate that the asymptotic behaviour is approached very slowly. Thus the numerical evidence may not be particularly illuminating.

2. An explicit formula. In proving our Theorem we require the following formula, which relates zeros of $\zeta(s)$ to prime numbers.

LEMMA. If $1<\sigma<2$ and $x \geqq 1$ then

$$
\begin{aligned}
(2 \sigma-1) \sum_{\gamma} \frac{x^{i \gamma}}{\left(\sigma-\frac{1}{2}\right)^{2}+(t-\gamma)^{2}}= & -x^{-1 / 2}\left(\sum_{n \leqq x} \Lambda(n)\left(\frac{x}{n}\right)^{1-\sigma+i t}+\sum_{n>x} \Lambda(n)\left(\frac{x}{n}\right)^{\sigma+i t}\right) \\
& +x^{1 / 2-\sigma+i t}\left(\log \tau+O_{\sigma}(1)\right)+O_{\sigma}\left(x^{1 / 2} \tau^{-1}\right),
\end{aligned}
$$

where $\tau=|t|+2$. The implicit constants depend only on $\sigma$.

Proof. It is well known (see [2, p. 353]) that if $x>1, x \neq p^{n}$, then

$$
\sum_{n \leqq x} \Lambda(n) n^{-s}=-\frac{\zeta^{\prime}}{\zeta}(s)+\frac{x^{1-s}}{1-s}-\sum_{Q} \frac{x^{\varrho-s}}{\varrho-s}+\sum_{n=1}^{\infty} \frac{x^{-2 n-s}}{2 n+s}
$$

provided $s \neq 1, s \neq \varrho, s \neq-2 n$. This does not depend on RH, but if we assume RH then the above may be expressed as 


$$
\sum_{\varrho} \frac{x^{i \gamma-i t}}{\sigma-\frac{1}{2}+i t-i \gamma}=x^{\sigma-1 / 2}\left(\frac{\zeta^{\prime}}{\zeta}(s)+\sum_{n \leqq x} A(n) n^{-s}-\frac{x^{1-s}}{1-s}-\sum_{n=1}^{\infty} \frac{x^{-2 n-s}}{2 n+s}\right) .
$$

If we replace $s$ by $1-\sigma+i t$ in the above then we have

$$
\begin{aligned}
\sum_{\varrho} \frac{x^{i \gamma-i t}}{\frac{1}{2}-\sigma+i t-i \gamma}=x^{1 / 2-\sigma}\left(\frac{\zeta^{\prime}}{\zeta}(1-\sigma+i t)\right. & +\sum_{n \leqq x} A(n) n^{\sigma-1-i t} \\
& \left.-\frac{x^{\sigma-i t}}{\sigma-i t}-\sum_{n=1}^{\infty} \frac{x^{-2 n-1+\sigma-i t}}{2 n+1-\sigma+i t}\right) .
\end{aligned}
$$

We subtract respective sides of (20) from (19), and use the relation

$$
\frac{\zeta^{\prime}}{\zeta}(s)=-\sum_{n=1}^{\infty} \Lambda(n) n^{-s}
$$

which holds for $\sigma>1$. We find that

$$
(2 \sigma-1) \sum_{Q} \frac{x^{i \gamma}}{\left(\sigma-\frac{1}{2}\right)^{2}+(t-\gamma)^{2}}=-x^{-1 / 2}\left(\sum_{n \leqq x} \Lambda(n)\left(\frac{x}{n}\right)^{1-\sigma+i t}+\sum_{n>x} \Lambda(n)\left(\frac{x}{n}\right)^{\sigma+i t}\right)
$$

$$
\begin{aligned}
& -\frac{\zeta^{\prime}}{\zeta}(1-\sigma+i t) x^{1 / 2-\sigma+i t}+\frac{x^{1 / 2}(2 \sigma-1)}{(\sigma-1+i t)(\sigma-i t)} \\
& -x^{-1 / 2} \sum_{n=1}^{\infty} \frac{(2 \sigma-1) x^{-2 n}}{(\sigma-1-i t-2 n)(\sigma+i t+2 n)} .
\end{aligned}
$$

Both sides of the above are continuous for all $x \geqq 1$, so we no longer exclude the values $x=1, x=p^{n}$. If $1<\sigma<2$, then from the logarithmic derivative of the functional equation of the zeta function (see [1, pp. 75, 82-83]) we have

$$
\frac{\zeta^{\prime}}{\zeta}(1-\sigma+i t)=-\frac{\zeta^{\prime}}{\zeta}(\sigma-i t)-\log \tau+O_{\sigma}(1)
$$

from (21) we see that this is $=-\log \tau+O_{\sigma}(1)$. Hence the right-hand side of (22) is

$$
\begin{aligned}
= & -x^{-1 / 2}\left(\sum_{n \leqq x} \Lambda(n)\left(\frac{x}{n}\right)^{1-\sigma+i t}+\sum_{n>x} \Lambda(n)\left(\frac{x}{n}\right)^{\sigma+i t}\right) \\
& +x^{1 / 2-\sigma+i t}\left(\log \tau+O_{\sigma}(1)\right)+O_{\sigma}\left(x^{1 / 2} \tau^{-2}\right)+O_{\sigma}\left(x^{-2} \tau^{-1}\right),
\end{aligned}
$$

which gives the result. 
3. Proof of the Theorem. The first assertion of the Theorem follows from the observation that we may interchange $\gamma$ and $\gamma^{\prime}$ in (1). To prove the remaining assertions, take $\sigma=\frac{3}{2}$ in the Lemma, and write (18) briefly as $L(x, t)=R(x, t)$. We evaluate the integrals $\int_{0}^{T}|L(x, t)|^{2} d t, \int_{0}^{T}|R(x, t)|^{2} d t$.

We treat the left-hand side first. We have

$$
\int_{0}^{T}|L(x, t)|^{2} d t=4 \sum_{\gamma, \gamma^{\prime}} x^{i\left(\gamma-\gamma^{\prime}\right)} \int_{0}^{T} \frac{d t}{\left(1+(t-\gamma)^{2}\right)\left(1+\left(t-\gamma^{\prime}\right)^{2}\right)} .
$$

We wish to exclude those numbers $\gamma \notin[0, T]$. It suffices to show that

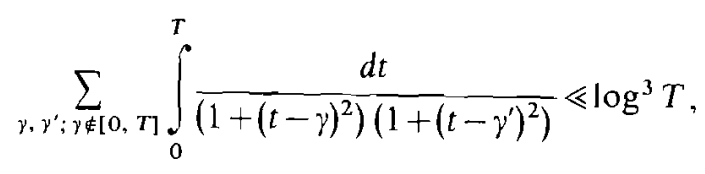

for then (23) is

$$
=4 \sum_{0<\gamma \leqq T ; 0<\gamma^{\prime} \leqq T} x^{i\left(\gamma-\gamma^{\prime}\right)} \int_{0}^{T} \frac{d t}{\left(1+(t-\gamma)^{2}\right)\left(1+\left(t-\gamma^{\prime}\right)^{2}\right)}+O\left(\log ^{3} T\right) .
$$

To prove (24) we use the fact (Theorem 9.2 of $[8]$ ) that if $T \geqq 2$ then there are $\ll \log T$ zeros for which $T \leqq \gamma \leqq T+1$. From this it is immediate that if $0 \leqq t \leqq T$ then

$$
\sum_{\gamma: \gamma \notin[0, T]} \frac{1}{1+(t-\gamma)^{2}} \ll\left(\frac{1}{t+1}+\frac{1}{T-t+1}\right) \log T
$$

and

$$
\sum_{\gamma^{\prime}} \frac{1}{1+\left(t-\gamma^{\prime}\right)^{2}} \ll \log T
$$

On the left-hand side of $(24)$ we take the sums inside and use the above estimates. The integration is then trivial, and we obtain (24).

Arguing similarly we may also show that

$$
\sum_{0<\gamma \leqq T ; 0<\gamma^{\prime} \leqq T} \int_{T}^{\infty} \frac{d t}{\left(1+(t-\gamma)^{2}\right)\left(1+\left(t-\gamma^{\prime}\right)^{2}\right)} \ll \log ^{2} T \int_{T}^{\infty} \frac{d t}{(t-T+1)^{2}} \ll \log ^{2} T .
$$


The estimation of $\sum_{0<\gamma \leqq T ; 0<\gamma^{\prime} \leqq T} \int_{-\infty}^{0} \ldots$ is the same, so we see that $(25)$ is

$$
=4 \sum_{0<\gamma \leqq T ; 0<\gamma^{\prime} \leqq T} x^{i\left(\gamma-\gamma^{\prime}\right)} \int_{-\infty}^{+\infty} \frac{d t}{\left(1+(t-\gamma)^{2}\right)\left(1+\left(t-\gamma^{\prime}\right)^{2}\right)}+O\left(\log ^{3} T\right) .
$$

From the calculus of residues we deduce that the definite integral is $=(\pi / 2) w\left(\gamma-\gamma^{\prime}\right)$, so the above is

$$
=2 \pi \sum_{0<\gamma \leqq T ; 0<\gamma^{\prime} \leqq T} x^{i\left(\gamma-\gamma^{\prime}\right)} w\left(\gamma-\gamma^{\prime}\right)+O\left(\log ^{3} T\right) .
$$

If we put $x=T^{\alpha}$ then we have

$$
\int_{0}^{T}\left|L\left(T^{\alpha}, t\right)\right|^{2} d t=F(\alpha) T \log T+O\left(\log ^{3} T\right)
$$

Here the left-hand side is clearly nonnegative, so we have the second assertion of the Theorem.

To complete the proof of the Theorem we prove (2); to this end we evaluate $\int_{0}^{T}|R(x, t)|^{2} d t$. In the first place

$$
\int_{0}^{T}\left|x^{-1+i t} \log \tau\right|^{2} d t=\frac{T}{x^{2}}\left(\log ^{2} T+O(\log T)\right)
$$

for all $x \geqq 1, T \geqq 2$. To compute the mean square of the Dirichlet series on the right-hand side of (18) we use the following quantitative form (see [5]) of Parseval's identity for Dirichlet series:

$$
\int_{0}^{T}\left|\sum_{n} a_{n} n^{-i t}\right|^{2} d t=\sum_{n}\left|a_{n}\right|^{2}(T+O(n))
$$

We could instead use the weaker relation

$$
\int_{0}^{T}\left|\sum_{n \leqq N} a_{n} n^{-i t}\right|^{2} d t=(T+O(N)) \sum_{n \leqq N}\left|a_{n}\right|^{2}
$$


this is Theorem 1.6 of [4]. However, the latter is restricted to Dirichlet polynomials, so we simplify our treatment by arguing from (28). We have

$$
\begin{aligned}
\frac{1}{x} \int_{0}^{T} \mid \sum_{n \leqq x} A(n)\left(\frac{x}{n}\right)^{-1 / 2+i t} & +\left.\sum_{n>x} \Lambda(n)\left(\frac{x}{n}\right)^{3 / 2+i t}\right|^{2} d t \\
& =\frac{1}{x} \sum_{n \leqq x} A(n)^{2}\left(\frac{x}{n}\right)^{-1}(T+O(n))+\frac{1}{x} \sum_{n>x} A(n)^{2}\left(\frac{x}{n}\right)^{3}(T+O(n)) .
\end{aligned}
$$

By the prime number theorem with error term this is

$$
=T(\log x+O(1))+O(x \log x) .
$$

As for the error terms in (18), we see that

$$
\int_{0}^{T}\left|x^{-1+i t}\right|^{2} d t=\frac{T}{x^{2}},
$$

and

$$
\int_{0}^{T} x \tau^{-2} d t \ll x
$$

We now combine our estimates (27), (29), (30), (31); we employ the following consequence of the Cauchy-Schwarz inequality: If $M_{k}=\int_{0}^{T}\left|A_{k}(t)\right|^{2} d t$ and $M_{1} \geqq M_{2}$ $\geqq M_{3} \geqq M_{4}$, then

$$
\int_{0}^{T}\left|\sum_{k=1}^{4} A_{k}(t)\right|^{2} d t=M_{1}+O\left(\left(M_{1} M_{2}\right)^{1 / 2}\right) .
$$

We consider three cases.

Case $1.1 \leqq x \leqq(\log T)^{3 / 4}$. Then our $M_{1}$ term is given by (27). Our other terms are uniformly $o\left(M_{1}\right)$, so our expression is $=(1+o(1))\left(T / x^{2}\right) \log ^{2} T$.

Case 2. $(\log T)^{3 / 4}<x \leqq(\log T)^{3 / 2}$. In this case all our estimates are uniformly $o(T \log T)$.

Case 3. $(\log T)^{3 / 2}<x \leqq T / \log T$. Then our $M_{1}$ term is given by (29). All our 
other terms are uniformly $o\left(M_{1}\right)$, so our expression is $=(1+o(1)) T \log x$.

If we put $x=T^{\alpha}$ then we may express our result by saying that

$$
\int_{0}^{r}\left|R\left(T^{\alpha}, t\right)\right|^{2} d t=\left((1+o(1)) T^{-2 \alpha} \log T+\alpha+o(1)\right) T \log T,
$$

uniformly for $0 \leqq \alpha \leqq 1-\varepsilon$. This and (26) give (2), so the proof is complete.

If $\alpha>1$ in the above then $x>T$, so the second error term in (29) is no longer smaller than the main term. The error term (31) also gives problems; a little consideration reveals that what we require is to know the size of

$$
\int_{0}^{r}\left|\frac{1}{x} \sum_{n \leqq x} \Lambda(n) n^{1 / 2-i t}+x \sum_{n>x} \Lambda(n) n^{-3 / 2-i t}-\frac{2 x^{1 / 2-i t}}{\left(\frac{1}{2}+i t\right)\left(\frac{3}{2}-i t\right)}\right|^{2} d t .
$$

If we multiply out the integrand and integrate terms individually, we find that there are too many nondiagonal terms to be ignored. We may, however, collect terms so that the above is expressed in terms of sums of the sort $\sum_{n \leqq y} \Lambda(n) \Lambda(n+h)$. There are various indications that this sum is approximately $c(h) y$, where $c(h)$ is a certain arithmetic constant. If we replace these sums by their conjectured approximations $c(h) y$, then our new expression is $\sim T \log T$. Moreover, there is a reasonable hypothesis as to the size of the differences

$$
\sum_{n \leqq y} \Lambda(n) \Lambda(n+h)-c(h) y
$$

which if true would allow us to carry out our program for $1 \leqq \alpha<2$. If the differences (33) are not only reasonably small but also behave independently for different $h$ then (32) is $\sim T \log T$ for all $\alpha \geqq 1$.

Another indication of the behaviour of the expression (32) can be obtained by considering its " $q$-analogue." The expression

$$
\sum_{q \leqq Q} \frac{1}{\varphi(q)} \sum_{\chi \neq \chi_{0}}\left|\frac{1}{x} \sum_{n \leqq x} \Lambda(n) \chi(n) n^{1 / 2}+\sum_{n>x} \Lambda(n) \chi(n) n^{-3 / 2}\right|^{2}
$$

may be shown to be $\sim Q \log x$ for $Q \geqq x$, in analogy with (29). If $x(\log x)^{-A} \leqq Q \leqq x$ then we may use an established technique [4, Chapter 17] to show that (34) is $\sim Q \log Q$. If $\mathrm{GRH}$ is true then this latter asymptotic relationship holds for $x^{3 / 4+\varepsilon}<Q \leqq x$. This corresponds to $1 \leqq \alpha<\frac{4}{3}$. One does not expect a change in the 
behaviour for larger $\alpha$, but a more delicate error-term analysis is needed if the result is to be extended.

4. The corollaries. To prove Corollary 1 we use our Theorem in conjunction with (3). To obtain (5) we take $r(u)=(\sin 2 \pi \alpha u) / 2 \pi \alpha u$. The Theorem makes it a simple task to compute

$$
\int_{-\infty}^{+\infty} F(\beta) \hat{r}(\beta) d \beta=\frac{1}{2 \alpha} \int_{-\alpha}^{\alpha} F(\beta) d \beta
$$

To obtain (6) we take $r(u)=((\sin \pi \alpha u) / \pi \alpha u)^{2}$. Again from the Theorem it is easy to compute

$$
\int_{-\infty}^{+\infty} F(\beta) \hat{r}(\beta) d \beta=\frac{1}{\alpha^{2}} \int_{-\alpha}^{+\alpha}(\alpha-\beta) F(\beta) d \beta
$$

We now prove Corollary 2 . Let $m_{e}$ be the multiplicity of the zero $\varrho$. In a sum over $0<\gamma \leqq T$, our convention concerning multiple zeros is that zeros are counted according to their multiplicities. This is accomplished by allowing $\gamma$ to take on the same value $m_{\varrho}$ times. In particular,

$$
\sum_{0<\gamma \leqq T} m_{e}=\sum_{\substack{0<\gamma \leq T \\ 0<\gamma^{\prime} \doteq T \\ \gamma=\gamma^{\prime}}} 1
$$

for on both sides a given zero $\varrho$ is counted with weight $m_{Q}^{2}$. But

$$
\sum_{\substack{0<\gamma \leqq T \\ 0<\gamma^{\prime} \leqq T \\ \gamma=\gamma^{\prime}}} 1 \leqq \sum_{0<\gamma \leqq T ; 0<\gamma^{\prime} \leqq T}\left(\frac{\sin (\alpha / 2)\left(\gamma-\gamma^{\prime}\right) \log T}{(\alpha / 2)\left(\gamma-\gamma^{\prime}\right) \log T}\right)^{2} w\left(\gamma-\gamma^{\prime}\right)
$$

and if we take $\alpha=1-\delta$ then from (6) the above is

$$
\leqq\left(\frac{4}{3}+\varepsilon\right)(T / 2 \pi) \log T \text {. }
$$

Hence we have demonstrated that

$$
\sum_{0<y \leqq T} m_{e} \leqq\left(\frac{4}{3}+o(1)\right)(T / 2 \pi) \log T .
$$


Now

$$
\sum_{0<\gamma \leqq T ; \rho \text { simple }} 1 \geqq \sum_{0<\gamma \leqq T}\left(2-m_{\varrho}\right) \geqq\left(2-\frac{4}{3}+o(1)\right) \frac{T}{2 \pi} \log T,
$$

so we have Corollary 2 . The kernel $\hat{r}(u)$ which we have used does not appear to be optimal for our purpose, so presumably one can improve slightly on the constant $\frac{2}{3}$.

We now turn to the first assertion of Corollary 3. We take $r(u)=$ $\max (1-(|u| / \lambda), 0)$ in (3), and choose $\lambda$ later. Now $\hat{r}(\alpha)$ is nonnegative, and $\int_{0}^{\infty} \hat{r}(\alpha) d \alpha<\infty$, so our Theorem permits us to calculate a lower bound for the right-hand side of (3). We see that

$$
\int_{-\infty}^{+\infty} F(\alpha) \hat{r}(\alpha) d \alpha \geqq(1+o(1))\left(\lambda+2 \lambda \int_{0}^{1} \alpha\left(\frac{\sin \pi \lambda \alpha}{\pi \lambda \alpha}\right) d \alpha\right) \frac{T}{2 \pi} \log T .
$$

We may assume that all but finitely many zeros are simple, so the terms $\gamma=\gamma^{\prime}$ in (3) contribute an amount $\sim(T / 2 \pi) \log T$. Hence

$$
\sum_{\substack{0<\gamma \leqq T \\ 0<\gamma^{\prime} \leqq T \\<\gamma-\gamma^{\prime}<2 \pi \lambda / \log T}} 1 \geqq\left(\frac{1}{2}+o(1)\right) C(\lambda) \frac{T}{2 \pi} \log T
$$

where

$$
C(\lambda)=\lambda+\left(1 / \pi^{2} \lambda\right) \operatorname{Cin}(2 \pi \lambda)-1
$$

Here $\operatorname{Cin}(x)$ is the "cosine integral,"

$$
\operatorname{Cin} x=\int_{0}^{x} \frac{1-\cos u}{u} d u .
$$

Note that the integrand is nonnegative, so that $\operatorname{Cin}(x)>0$ for $x>0$. To obtain (8) we show that $C(\lambda)>0$ for some $\lambda<1$. This is easy, because $C(1)=\left(1 / \pi^{2}\right)$ - $\operatorname{Cin}(2 \pi)>0$, and $C(\lambda)$ is continuous. In fact a little calculation reveals that $C(0.68)$ $>0$. We have not determined the optimal kernel $\hat{r}(\alpha)$, so one should be able to improve on the constant 0.68 . 


\section{REFERENCES}

1. H. Davenport, Multiplicative number theory, Lectures in Advanced Math., no. I, Markham, Chicago, Ill., 1967. MR 36 \# 117.

2. Edmund Landau, Handbuch der Lehre von der Verteilung der Primzahlen, Teubner, Berlin, 1909.

3. M. L. Mehta. Random matrices and the statistical theorv of energv levels, Academic Press, New York, 1967. MR 36 \#3554.

4. Hugh L. Montgomery, Topics in multiplicative number theory, Lecture Notes in Math., vol. 227, Springer-Verlag, Berlin and New York, 1971.

5. Hugh L. Montgomery and R. C. Vaughan, Hilbert's inequality (to appear).

6. I. B. Rosser, J. M. Yohe and L. Schoenfeld, Rigorous computation and the zeros of the Riemann zeta-function (with discussion), Information Processing 68 (Proc. IFIP Congress, Edinburgh, 1968), vol. 1, Math., Software, North-Holland, Amsterdam, 1969, pp. 70-76. MR 41 \#2892.

7. Atle Selberg, On the zeros of Riemann's zeta-function, Skr. Norske Vid. Akad. Oslo I (1942), no. 10,59 pp. MR 6, 58

8. E. C. Titchmarsh, The theory of the Riemann zeta-function. Clarendon Press, Oxford, $195 \mathrm{I}$. MR 13, 74l.

\section{Trinity College}

Cambridge, England 